\title{
Role of endoscopy in suspicion of atrophic gastritis with and without intestinal metaplasia in comparison to histopathology
}

\author{
H. Ibrahim', A. Shams El-Deen², Z.A. Kasemy³ ${ }^{3}$ M. Saad ${ }^{4}$, A.A. Sakr ${ }^{1}$
}

(1) Tropical Medicine Department, Faculty of Medicine, Menoufia University, Menoufia, Egypt ; (2) Pathology Department, Faculty of Medicine, Menoufia University, Menoufia, Egypt ; (3) Public Health and Community Medicine, Faculty of Medicine, Menoufia University, Menoufia, Egypt ; (4) Hepatology \&Gastroenterology Department, Shibin El-Kom Fever hospital, Menoufia, Egypt.

\begin{abstract}
Background and study aims : Atrophic gastritis (AG) and intestinal metaplasia (IM) are established premalignant gastric lesions. Many studies documented a poor correlation between esophagogastroduodenoscopy (EGD) and histopathological (HP) findings of precancerous gastric lesions. The aim was to bridge the gap between endoscopy and HP in detection of chronic gastritis, AG and IM.

Patients and methods : a prospective single-center study involved 150 patients with endoscopic criteria of gastric lesions with upper gastrointestinal symptoms referred for upper GI endoscopy met the endoscopic criteria and classified according to HP of biopsies from targeted gastric lesions into chronic gastritis (GI), AG(GII) or IM(GIII). We correlated the endoscopic criteria of the 3 groups with the HP results.

Results : (73males \& 75 females) with ages ranged17-75 years and mean \pm SD was $41.96 \pm 15.95$. GI, GII \& GIII were [42 patients $(28 \%), 82$ patients $(54.7 \%)$ and 26 patients $(17.3 \%)]$, respectively. Diffuse gastric mottling was more common in GI $(74.3 \%, P<0.001)$, visible submucosal vessels, gastric atrophy predominated in GII (75.6, 82.3 \& 73.1\% $(P$ 0.005,0.4 \& $<0.01)$ ), respectively. Whitish raised lesions were more specific in GIII $(85.7 \%)(P<0.001)$. The sensitivity and specificity of endoscopic suspicion of chronic gastritis were $(86 \& 88 \%$ in GI), $(87 \& 85 \%$ in GII) and $(54 \% \& 100 \%$ in GIII) (p-0.001). The logistic regression model for risk factors was $\chi 2=25.74$ and 49.32, $p<0.001$.

Conclusion: Conventional endoscopy has high sensitivity and specificity for suspicion of chronic gastritis and AG, but low sensitivity and very high specificity for IM. Targeted biopsies may be valuable with image enhanced techniques. (Acta gastroenterol. belg., 2021, 84, 9-17).
\end{abstract}

Keywords : endoscopy, atrophic gastritis, histopathology, intestinal metaplasia.

\section{Introduction}

Atrophic gastritis (AG) and intestinal metaplasia (IM) are established premalignant gastric lesions (1). Incidence of gastric cancer (GC) is the 5th among cancers worldwide with more than $95 \%$ are adenocarcinomas (2) and the 3rd cause of cancer-related deaths worldwide (3). Recently, H. pylori infects more than half of the population in developing countries leading to a sequelae of chronic gastritis, AG, IM, and finally invasive gastric carcinoma in untreated patients $(4,5)$.

However, recent studies suggest that eradication of $H$. pylori infection reduces the incidence of its complications especially GC to about $33-47 \%$, other trials documented the development of AG, IM, and GC in previously eradicated patients (5).
Clinically, AG and IM are difficult to be detected as they have no specific symptoms or signs with most cases discovered endoscopically and or by histopathological assessment (3).

AG is a chronic inflammatory condition that results from a decrease or loss of appropriate gastric glands ; however, IM is the replacement of normal gastric glands by small intestinal phenotype metaplastic ones; the pathogenesis of which remains unclear. A systematic review reported recently a range of $0.53-15.24 / 1000$ person/year with AG and 0.38-17.08/1000 person with IM lead to GC annually (6). A large study done on 61707 patients with gastric IM, revealed annual progression to GC by $0.25 \%$ (3). Moreover, IM increases the risk of GC by 6 -folds (2).

Pathologically, IM can be identified by simple columnar epithelium containing paneth, absorptive, and goblet cells (3).

Recent European and international Kyoto guidelines highly recommend endoscopic surveillance of patients with extensive AG and IM at a 3-years interval (6).

Endoscopic detection of AG and IM had been missed for long periods until the revolutionary development of methylene blue staining; a type of chromo endoscopy by Ida et al., (1975) passing through Indigo carmine dye which allowed good visualization of mucosal irregularities and atypical vascular pattern characteristic of IM. However, recently, the great advance in endoscopic technology with the detection of image enhanced endoscopy (IEE) as Narrow Band Imaging (NBI) has increased the detection rate of mucosal abnormalities. Practically, most endoscopists still use the conventional white-light endoscopy (CWLE) and High-definition (HD-WLE) in daily practice for endoscopic diagnosis of chronic gastritis, $\mathrm{AG}$, and $\mathrm{IM}$ due to insufficient experience and /or shortage of resources (6).

Moreover, chronic gastritis and AG have been diagnosed by histopathological analysis for long periods with poor correlation with the endoscopic findings.

Correspondence to : Ayman Ahmed Sakr, Lecturer of Tropical Medicine, Tropical Medicine Department, Faculty of Medicine, Yassine Abd Elghafar street, Shibin El-Kom, Menoufia, Egypt. Tel. : 02(1009870858), (81) 8049593365.

E-mail : aymanahmedsakr@gmail.com

Submission date : 25/02/2020

Acceptance date : $30 / 07 / 2020$ 
Recent advance in endoscopic technologies yields endoscopists to largely divert the diagnosis to the endoscopic rather than histological patterns. Endoscopic features that correlate well with histopathology become more evident with many studies done on this aspect (7). Additionally, this will decrease the biopsy costs and post-biopsy complications (8).

Successful eradication of $H$. pylori has been studied with a dramatic reduction in $H$. pylori-induced gastritis, atrophy, metaplasia, and finally GC. However, there is still a debate on post-eradication follow up.

The work aimed to explore endoscopic-histopathological correlation based mainly on white light endoscopy used in routine practice in tertiary centers in Egypt. Additionally, we need to evaluate the effect of treatment or eradication of H.pylori on the development of AG and IM.

\section{Patients and methods}

\section{Patients}

This was a prospective single-center study involved 150 patients with endoscopic criteria of chronic gastritis, AG or IM selected from 300 patients with upper gastrointestinal symptoms who were referred to GI endoscopy units, Tropical Medicine Department, Menoufia university tertiary hospitals for upper GI endoscopy in the period between August 2017 to August 2018. They were 73 males $(48.7 \%)$ and 77 females $(51.3 \%)$ and their ages ranged between 17 and 75 years with mean value $(41.96 \pm 15.95$ years $)$.

All patients enrolled in this project were complaining of upper gastrointestinal symptoms including dyspepsia, nausea, vomiting, upper gastrointestinal bleeding (UGIB), reflux symptoms, and anemia who were in need of esophagogastroduodenoscopy (EGD) evaluation.

Patients with previous gastric surgery, on protonpump inhibitor treatment, already diagnosed with GC or lymphoma, or had either a contraindication to undergo EGD or to have gastric biopsies or had esophageal or duodenal lesions as the main cause of their symptoms were excluded from the study (Figure 1). All related laboratory measures were done 24 hours prior to the endoscopic procedures.

\section{Endoscopic evaluation}

All patients were assessed with qualified endoscopists with more than 10 years of experience and performing more than 30 EGDs (diagnostic and therapeutic) per month and well-trained on basic and advanced endoscopic features suggesting chronic atrophic and non-atrophic gastritis, IM and early gastric cancer using CWLE, HDWLE, IEE.

Only Olympus scopes (Tokyo, Japan) models 170, 240, and 260 with conventional white light models (C-WLE) were used with fewer patients were examined using

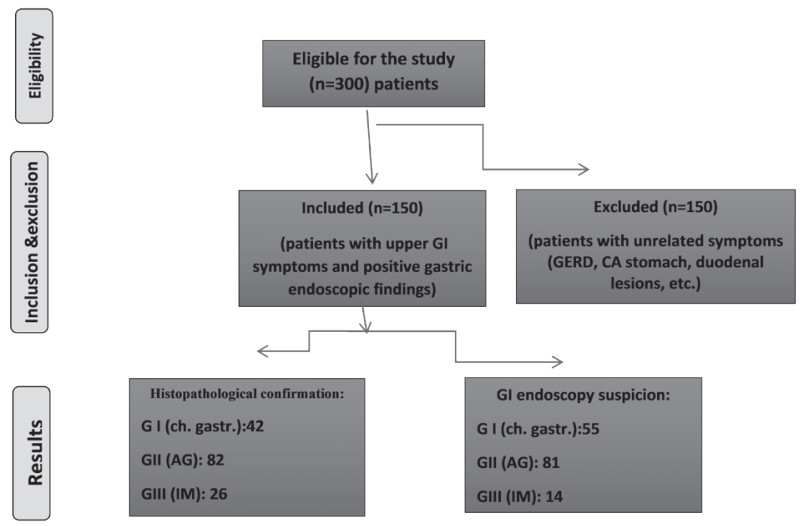

Figure 1. - Flow chart of the studied patients.

higher versions with a high -definition (HD-WLE) and image-enhanced techniques 260,170 \&190 with narrowband imaging (NBI).

The studied patients were prepared for EGD with overnight fasting. EGD was done down to the $2^{\text {nd }}$ part of the duodenum with a complete exploration of the whole stomach including the blind areas. According to a recent European society of gastrointestinal endoscopy guidelines for performance measures of upper gastrointestinal endoscopy, we stressed on complete visualization of the whole gastric mucosa through the absence of any food particles, debris, or mucus and using water jet system. The average endoscopic examination was 10 minutes (9). Endoscopic findings were reported and standardized.

Gastric mucosal atrophy was diagnosed endoscopically by the visibility of vascular pattern and gastric rugae atrophy. While IM was detected as greyish-whitish slightly opaque patches using CWLE or as a light blue crest (LBC), or white opaque substance (WOS) using NBI (7).

Endoscopic features that can characterize H. pylori infection were erythema, mucosal swelling, nodularity, streaks, diffuse or localized erythemas or spotty areas. Mucosal swelling is a soft thick edematous mucosa. A normal gastric mucosal fold is about $5 \mathrm{~mm}$, smooth and straight. Enlarged fold was considered if more than $5 \mathrm{~mm}$ in thickness and tortuous. Biopsies were taken from suspicious gastric lesions under CWLE. In order to diagnose chronic H.pylori active gastritis and in case of a diffuse gastric pathology as diffuse or spotty redness, nodularity or mottling, biopsies had been taken according to updated Sydney system with 2 biopsies from antrum, one from incisura, one from lesser curvature and the $5^{\text {th }}$ from greater curvature each in a separate tube (9).

All the obtained biopsies were collected for histopathology (HP), fixed in 10\% neutral formalin, and sent for preparation of formalin-fixed, paraffin-embedded tissue blocks. 4 um thick sections were cut. One set of tissue sections was stained with hematoxylin and eosin for routine analysis and another set with Giemsa stain for detection of $H$. pylori infection in the gastric mucosa (10). 


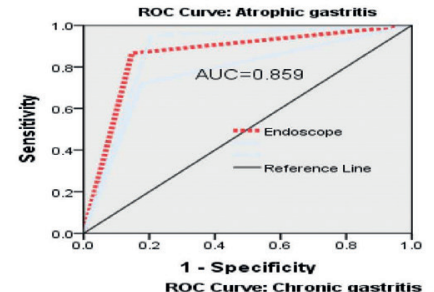

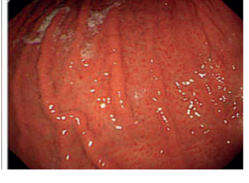

Patch redness (HD)

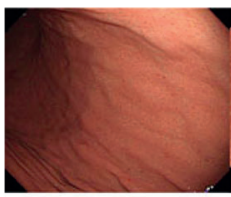

Diffuse redness (HD)

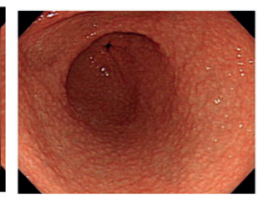

A) Endoscopic findings commonly detected in H.pylori related chronic gastritis CWLE



Thin atrophic mucosa
and rugae of the antrum

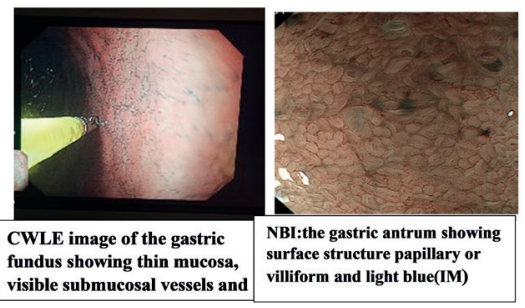

B) Endoscopic findings of AG using CWLE \& HD-WLE and NBI(IM).

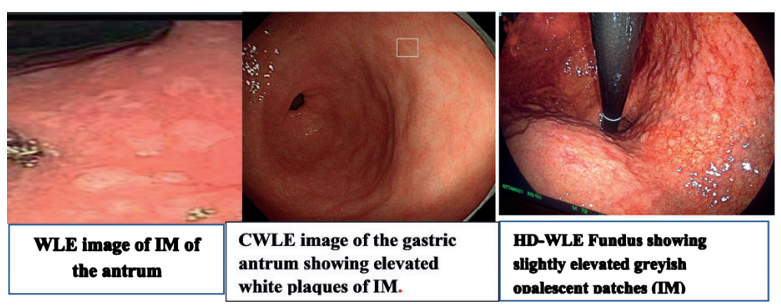

C) Endoscopic findings of IM using CWLE and HD-WLE

Figure 4. - Endoscopic views of IM and AG using CWLE and NBI.

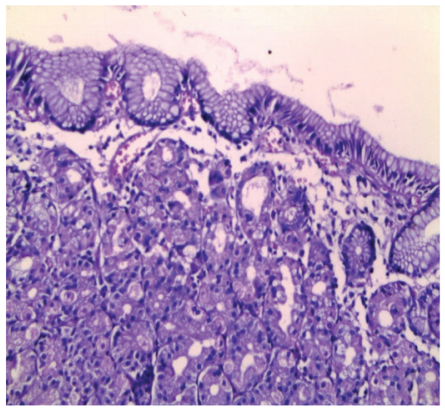

A case of $\mathrm{H}$ pylori associated chronic gastriti showing intestinal metaplasia with evident goblet cells in mucosal glands (H\&Ex200).

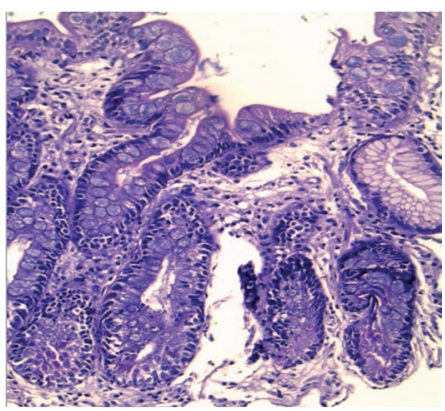

Another case of $\mathrm{H}$ pylori associated chronic gastritis showing marked atrophy of mucosal glands(H\&Ex100).

Figure 5. - Histopathological findings in two cases with IM and AG.
Then, we correlated the endoscopic findings with the histopathological results in all groups. 
Patients were classified into three groups

G I : patients with histopathological confirmation of chronic non-atrophic gastritis $(\mathrm{No}=42)$.

G II : patients with histopathological confirmation of chronic AG $(\mathrm{No}=82)$.

G III : patients with histopathological confirmation of $\operatorname{IM}(\mathrm{No}=26)$.

Frequency data were summarized as percentages. continuous variables were summarized as median and range of distribution. A two-sided $\mathrm{P}<0.05$ means the null hypothesis rejection character. 95\% CI methods for OR was based on mid P-method. Logistic regression is a statistical technique used to predict the probability of binary response based on one or more independent variables. The SPSS 20.0 (SPSS Inc, IBM Corporation, Armonk, NY, USA) software was used for statistical analysis. This project was approved by Menoufia university ethical board.

\section{Results}

In a total, 300 patients were referred for EGD evaluation due to their upper gastrointestinal symptoms, 150 patients met the endoscopic criteria of chronic gastritis, AG or,
IM. All other patients were excluded .73 were males and 77 were females with ages ranged from 17-75 years; mean \pm SD (41.96 \pm 15.95$)$. Chronic gastritis (GI), AG (GII) \& IM (GIII) were (42 patients (28\%), 82 patients $(54.7 \%)$ and 26 patients $17.3 \%$ ), respectively (Figure 1). Smokers constitutes 67 patients (44.7\%), while diabetics were $69(46 \%)$. Patients with a history of previous $H$. pylori infection were 95 patients $(63.6 \%), 55 \%$ of them had a history of anti- $H$. pylori therapy with the family history of $H$. pylori infection was 55(36.6\%) (Table 1).

There was a statistically significant difference between GI and GIII regarding smoking, current and family history of H.pylori infection $(\mathrm{P}<0.001)$, GI and GII regarding chronic NSAIDs use, smoking and DM $(\mathrm{P}<0.001)$ and between GII and GIII regarding family history of H.pylori infection with a higher incidence of IM in patients who had a positive family history of H.pylori infection $(\mathrm{p}<0.001)$ (Table 4).

A logistic regression was performed to ascertain the effects of smoking, DM, H.pylori, HB, WBCs, and platelets on the likelihood that participants have AG or IM. The logistic regression model was statistically significant, $\chi 2=25.74$ and 49.32 respectively, $\mathrm{p}<0.001$. The model explained 61.0 and $70 \%$ (Nagelkerke R2) of the variance in AG or IM and correctly classified 86.3,

Table 1. - Clinical characteristics of the studied patients

\begin{tabular}{|c|c|c|}
\hline Clinical characteristics & \multicolumn{2}{|c|}{$\begin{array}{l}\text { Studied patients } \\
\quad(n=150)\end{array}$} \\
\hline $\begin{array}{l}\text { Age (Y) } \\
\text { - Mean } \pm \text { SD } \\
\text { - Range }\end{array}$ & \multicolumn{2}{|c|}{$\begin{array}{c}41.96 \pm 15.95 \\
17-75\end{array}$} \\
\hline \multirow{2}{*}{$\begin{array}{ll}\text { Sex } & \\
\text { - Male } \\
\text { - Female }\end{array}$} & No & $\%$ \\
\hline & $\begin{array}{l}73 \\
77\end{array}$ & $\begin{array}{l}48.7 \\
51.3\end{array}$ \\
\hline Smoking & 67 & 44.7 \\
\hline DM & 69 & 46.0 \\
\hline $\begin{array}{l}\text { H. Pylori infection: } \\
\text { - Current H.Pylori infection } \\
\text { - Previous H.Pylori therapy and it's timing }(\mathrm{no}=55) \text { : } \\
\quad<60 \\
>60 \\
\text { - Family history of H.Pylori infection }\end{array}$ & $\begin{array}{l}95 \\
24 \\
31 \\
55\end{array}$ & $\begin{array}{l}63.6 \\
\\
43.6 \\
56.4 \\
36.6\end{array}$ \\
\hline Family history of cancer stomach & 10 & 6.7 \\
\hline History of chronic use of NSAIDs & 26 & 17.3 \\
\hline $\begin{array}{l}\text { General examination: } \\
\text { - Pallor }\end{array}$ & 29 & 19.3 \\
\hline $\begin{array}{l}\text { Indications for EGD: } \\
\text { - Abdominal pain (persistent or recurrent) } \\
\text { - Dyspepsia } \\
\text { - Vomiting } \\
\text { - Unexplained anemia } \\
\text { - Heartburn } \\
\text { - Dysphagia } \\
\text { - GI bleeding (melena \& hematemesis) }\end{array}$ & $\begin{array}{l}122 \\
75 \\
30 \\
28 \\
24 \\
12 \\
12\end{array}$ & $\begin{array}{l}81.3 \\
50.0 \\
20.0 \\
18.7 \\
16.0 \\
8.0 \\
8.0\end{array}$ \\
\hline $\begin{array}{l}\text { Site of gastric biopsy } \\
\text { - Antrum } \\
\text { - Fundus } \\
\text { - Corpus }\end{array}$ & $\begin{array}{l}92 \\
35 \\
23\end{array}$ & $\begin{array}{l}61.3 \\
23.3 \\
15.3\end{array}$ \\
\hline \multirow{2}{*}{$\begin{array}{l}\text { Laboratory investigations } \\
\text { - } \mathrm{Hb}(\mathrm{g} / \mathrm{dl}) \\
\text { - WBCs }\left(\ldots \ldots \times 10^{3}\right) \\
\text { - Platelets }\left(\ldots \ldots \times 10^{3}\right)\end{array}$} & Mean \pm SD & Range \\
\hline & $\begin{array}{c}10.57 \pm 1.63 \\
6.37 \pm 1.96 \\
221.92 \pm 122.24\end{array}$ & $\begin{array}{c}7.90-14.0 \\
3-11.0 \\
49-498.0\end{array}$ \\
\hline
\end{tabular}


Table 2. - Distribution of endoscopic findings among the studied groups

\begin{tabular}{|c|c|c|c|c|c|c|c|c|}
\hline \multirow{3}{*}{ Endoscopic Findings } & \multicolumn{6}{|c|}{ Endoscopic evidence } & \multirow{3}{*}{$\chi^{2}$} & \multirow{3}{*}{$\mathbf{P}$} \\
\hline & \multicolumn{2}{|c|}{$\begin{array}{l}\text { Chronic gastritis } \\
(\mathrm{N}=42)\end{array}$} & \multicolumn{2}{|c|}{$\begin{array}{l}\text { Atrophic gastritis } \\
\qquad(\mathrm{N}=\mathbf{8 2})\end{array}$} & \multicolumn{2}{|c|}{$\begin{array}{c}\text { Metaplasia } \\
(\mathbf{N}=\mathbf{2 6})\end{array}$} & & \\
\hline & No. & $\%$ & No. & $\%$ & No. & $\%$ & & \\
\hline Diffuse redness & 42 & 43.7 & 44 & 45.8 & 10 & 10.4 & 34.79 & $<0.001^{*}$ \\
\hline Diffuse mottling & 29 & 74.3 & 7 & 17.9 & 3 & 7.6 & 45.28 & $<0.001 *$ \\
\hline Antral granularity & 20 & 57.1 & 10 & 28.5 & 5 & 14.2 & 19.78 & $<0.001 *$ \\
\hline Enlarged gastric folds & 18 & 40.6 & 11 & 43.3 & 3 & 9.3 & 16.14 & $<0.001^{*}$ \\
\hline Antral erosions & 37 & 61.6 & 18 & 30.0 & 5 & 8.3 & 56.28 & $<0.001 *$ \\
\hline Fundal erosions & 5 & 55.5 & 2 & 22.2 & 2 & 22.2 & 4.57 & 0.101 \\
\hline Antral ulcer & 10 & 47.6 & 4 & 19.4 & 7 & 33.3 & 12.63 & $0.001 *$ \\
\hline Visible submucosal vessels & 5 & 12.2 & 31 & 75.6 & 5 & 12.2 & 10.42 & $0.005^{*}$ \\
\hline Atrophic mucosa Atrophic gastric folds & 1 & 5.8 & 14 & 82.3 & 2 & 11.7 & 6.38 & $0.041 *$ \\
\hline Whitish raised lesions & 5 & 12.1 & 30 & 73.1 & 6 & 14.6 & 8.80 & $0.012 *$ \\
\hline \multirow[t]{2}{*}{ Bile reflux } & 2 & 14.2 & 0 & 0.0 & 12 & 85.7 & 51.13 & $<0.001 *$ \\
\hline & 20 & 86.9 & 2 & 8.6 & 1 & 4.3 & 46.87 & $<0.001 *$ \\
\hline \multicolumn{7}{|l|}{ H.Pylori intensity } & \multirow{4}{*}{31.53} & \multirow{4}{*}{$<0.001$} \\
\hline Mild & 13 & 30.0 & 49 & 59.0 & 5 & 19.0 & & \\
\hline Moderate & 27 & 64.0 & 17 & 20.0 & 17 & 65.0 & & \\
\hline Severe & 2 & 4.0 & 16 & 19.0 & 4 & 15.0 & & \\
\hline
\end{tabular}

Table 3. - Sensitivity and specificity of CWLE in comparison to histopathology in the studied groups (A):

\begin{tabular}{|l|c|c|c|c|c|c|c|}
\hline \multicolumn{1}{|c|}{ Groups } & Sensitivity & Specificity & Accuracy & PPV & NPV & Kappa & P value \\
\hline Chronic gastritis & 86.0 & 88.0 & 87.0 & 73.0 & 94.0 & 0.70 & $<0.001 *$ \\
\hline Atrophic gastritis & 87.0 & 85.0 & 86.0 & 88.0 & 84.0 & 0.71 & $<0.001 *$ \\
\hline Intestinal metaplasia & 54.0 & 100.0 & 92.0 & 100.0 & 91.0 & 0.65 & $<0.001 *$ \\
\hline
\end{tabular}

(B): Sensitivity and specificity of HD-WLE with NBI in comparison to histopathology in the studied groups

\begin{tabular}{|c|c|c|c|c|c|c|c|}
\hline Groups & Sensitivity & Specificity & Accuracy & PPV & NPV & Kappa & P value \\
\hline Chronic gastritis & 86.0 & 88.0 & 95 & 58.17 & 77.22 & 0.77 & $<0.01$ \\
\hline Atrophic gastritis & 87.0 & 93 & 88 & 71.5 & 71.3 & 0.8 & $<0.01$ \\
\hline Intestinal metaplasia & 100 & 96 & 96 & 70 & 96.8 & 0.9 & $<0.05$ \\
\hline
\end{tabular}

Table 4. - Clinical characteristics of the studied groups

\begin{tabular}{|c|c|c|c|c|c|c|c|c|}
\hline & \multicolumn{6}{|c|}{ Groups } & \multirow{3}{*}{ Test of sig } & \multirow{3}{*}{ P-value } \\
\hline & \multicolumn{2}{|c|}{$\begin{array}{c}\text { Chronic gastritis } \\
(\mathrm{N}=42)\end{array}$} & \multicolumn{2}{|c|}{$\begin{array}{l}\text { Atrophic gastritis } \\
\qquad(\mathrm{N}=82)\end{array}$} & \multicolumn{2}{|c|}{$\begin{array}{l}\text { Intestinal metaplasia } \\
\qquad(\mathrm{N}=\mathbf{2 6})\end{array}$} & & \\
\hline & No & $\%$ & No & $\%$ & No & $\%$ & & \\
\hline $\begin{array}{l}\mathbf{H b} \\
\text { Mean } \pm \mathrm{SD}\end{array}$ & \multicolumn{2}{|c|}{$10.41 \pm 1.28$} & \multicolumn{2}{|c|}{$10.17 \pm 1.30$} & \multicolumn{2}{|c|}{$9.30 \pm 0.90$} & $\begin{array}{c}\text { Kruskal-Wallis }=11.55 \\
\mathrm{P}=0.001\end{array}$ & $\begin{array}{c}\mathrm{P} 2, \mathrm{P} 3 \\
<0.001^{*}\end{array}$ \\
\hline $\begin{array}{l}\text { WBCs } \\
\text { Mean } \pm \text { SD }\end{array}$ & \multicolumn{2}{|c|}{$6.67 \pm 1.19$} & \multicolumn{2}{|c|}{$6.55 \pm 2.04$} & \multicolumn{2}{|c|}{$6.16 \pm 1.90$} & $\begin{array}{l}\text { Kruskal-Wallis }=17.26 \\
\mathrm{P}<0.001^{*}\end{array}$ & $\begin{array}{l}\mathrm{P} 2, \mathrm{P} 3 \\
<0.05^{*}\end{array}$ \\
\hline $\begin{array}{l}\text { Platelets } \\
\text { Mean } \pm \text { SD }\end{array}$ & \multicolumn{2}{|c|}{$210.0 \pm 103.32$} & \multicolumn{2}{|c|}{$222.26 \pm 120.31$} & \multicolumn{2}{|c|}{$155.38 \pm 39.64$} & $\begin{array}{c}\text { Kruskal-Wallis }=4.08 \\
\mathrm{P}=0.043\end{array}$ & $\begin{array}{l}\mathrm{P} 2, \mathrm{P} 3 \\
<0.05^{*}\end{array}$ \\
\hline $\begin{array}{l}\text { Smoking } \\
\text { Yes } \\
\text { No }\end{array}$ & $\begin{array}{c}3 \\
39\end{array}$ & $\begin{array}{c}7.1 \\
92.9\end{array}$ & $\begin{array}{l}47 \\
35\end{array}$ & $\begin{array}{l}57.3 \\
42.7\end{array}$ & $\begin{array}{c}17 \\
9 \\
\end{array}$ & $\begin{array}{l}65.4 \\
34.6\end{array}$ & $\begin{array}{l}\chi^{2}=29.06 \\
\chi^{2}=26.24 \\
\chi^{2=}=0.53\end{array}$ & $\begin{array}{c}\mathrm{P} 1, \mathrm{P} 2<0.001 * \\
\mathrm{P} 3=0.465\end{array}$ \\
\hline $\begin{array}{l}\text { DM } \\
\text { Yes } \\
\text { No }\end{array}$ & $\begin{array}{l}24 \\
18\end{array}$ & $\begin{array}{l}57.1 \\
42.9\end{array}$ & $\begin{array}{l}30 \\
52\end{array}$ & $\begin{array}{l}36.6 \\
63.4\end{array}$ & $\begin{array}{l}15 \\
11\end{array}$ & $\begin{array}{c}57.7 \\
4.3\end{array}$ & $\begin{array}{c}\chi^{2}=4.78 \\
\chi^{2=}=0.0 \\
\chi^{2=} 3.62\end{array}$ & $\begin{array}{l}\mathrm{P} 1=0.028 * \\
\mathrm{P} 2=0.964 \\
\mathrm{P} 3=0.057\end{array}$ \\
\hline $\begin{array}{l}\text { Chronic use of NSAIDs } \\
\text { Yes } \\
\text { No }\end{array}$ & $\begin{array}{l}14 \\
28\end{array}$ & $\begin{array}{l}33.3 \\
66.7 \\
\end{array}$ & $\begin{array}{c}8 \\
74\end{array}$ & $\begin{array}{c}9.8 \\
90.2\end{array}$ & $\begin{array}{c}4 \\
22\end{array}$ & $\begin{array}{l}15.4 \\
84.6\end{array}$ & $\begin{array}{l}\chi^{2}=10.58 \\
\chi^{2}=2.66 \\
\chi^{2}=0.63\end{array}$ & $\begin{array}{l}\mathrm{P} 1<0.001 * \\
\mathrm{P} 2=0.103 \\
\mathrm{P} 3=0.426\end{array}$ \\
\hline $\begin{array}{l}\text { Current H. Pylori infection } \\
\text { Yes } \\
\text { No }\end{array}$ & $\begin{array}{l}20 \\
22\end{array}$ & $\begin{array}{l}47.6 \\
52.4\end{array}$ & $\begin{array}{l}52 \\
30\end{array}$ & $\begin{array}{l}63.4 \\
36.6\end{array}$ & $\begin{array}{c}23 \\
3\end{array}$ & $\begin{array}{l}88.5 \\
11.5\end{array}$ & $\begin{array}{c}\chi^{2}=2.85 \\
\chi^{2=} 11.52 \\
\chi^{2=}=5.84\end{array}$ & $\begin{array}{c}\mathrm{P} 1=0.091 \\
\mathrm{P} 2<0.001^{*} \\
\mathrm{P} 3=0.015^{*}\end{array}$ \\
\hline $\begin{array}{l}\text { Previous H. Pylori therapy } \\
\text { Yes } \\
\text { No }\end{array}$ & $\begin{array}{l}15 \\
27\end{array}$ & $\begin{array}{l}75.0 \\
25.0\end{array}$ & $\begin{array}{l}30 \\
52\end{array}$ & $\begin{array}{l}57.7 \\
42.3\end{array}$ & $\begin{array}{l}10 \\
16\end{array}$ & $\begin{array}{l}43.5 \\
56.5\end{array}$ & $\begin{array}{l}\chi^{2}=0.01 \\
\chi^{2=}=0.05 \\
\chi^{2}=0.03\end{array}$ & $\begin{array}{l}\mathrm{P} 1=0.923 \\
\mathrm{P} 2=0.819 \\
\mathrm{P} 3=0.862\end{array}$ \\
\hline $\begin{array}{l}\text { Family history of H. Pylori } \\
\text { infection } \\
\text { Yes } \\
\text { No }\end{array}$ & $\begin{array}{c}8 \\
34\end{array}$ & $\begin{array}{l}19.0 \\
81.0\end{array}$ & $\begin{array}{l}27 \\
55\end{array}$ & $\begin{array}{l}32.9 \\
67.1\end{array}$ & $\begin{array}{c}20 \\
6\end{array}$ & $\begin{array}{l}76.9 \\
23.1\end{array}$ & $\begin{array}{l}\chi^{2}=2.64 \\
\chi^{2}=22.21 \\
\chi^{2=} 15.55\end{array}$ & $\begin{array}{l}\mathrm{P} 1=0.104 \\
\mathrm{P} 2<0.001 * \\
\mathrm{P} 3<0.001 *\end{array}$ \\
\hline
\end{tabular}


Table 5. - Multivariate regression analysis of the studied groups

\begin{tabular}{|l|c|c|c|c|}
\hline \multirow{2}{*}{ Smoking } & \multicolumn{4}{|c|}{ Atrophic gastritis } \\
\cline { 2 - 5 } & Wald & Sig & OR & CI 95\% \\
\hline Diabetes mellitus & 18.30 & $<0.001^{*}$ & 36.72 & $7.04-191.37$ \\
\hline H. Pylori & 2.68 & 0.101 & 0.369 & $0.11-1.21$ \\
\hline Chronic use of NSAIDs & 6.60 & $0.010^{*}$ & 4.498 & $1.4-14.16$ \\
\hline Hb & $\mathbf{7 . 1 1}$ & 0.008 & 0.10 & $0.01-0.54$ \\
\hline WBCS & 1.46 & 0.26 & 0.778 & $0.51-1.16$ \\
\hline Platelets & 11.50 & 0.001 & 0.548 & $0.38-0.77$ \\
\hline & 0.18 & 0.669 & 0.999 & $0.99-1.0$ \\
\hline Smoking & \multicolumn{4}{|c|}{ Intestinal metaplasia } \\
\hline Diabetes mellitus & 6.41 & $0.011^{*}$ & 23.73 & $2.04-275.42$ \\
\hline H pylori & 0.93 & 0.335 & 2.94 & $0.32-26.53$ \\
\hline Chronic use of NSAIDs & 2.74 & 0.098 & 4.61 & $0.75-28.23$ \\
\hline Hb & 0.02 & 0.876 & 1.15 & $0.18-7.38$ \\
\hline WBCS & 2.41 & 0.120 & 0.53 & $0.23-1.18$ \\
\hline Platelets & 5.32 & 0.021 & 0.50 & $0.28-0.90$ \\
\hline
\end{tabular}

A logistic regression was performed to ascertain the effects of smoking, DM, H.pylori, Hb, WBCs and platelets on the likelihood that participants have atrophic gastritis or intestinal metaplasia. The logistic regression model was statistically significant, $\chi^{2}=25.74$ and 49.32 respectively, $p<0.001$. The model explained 61.0 and $70 \%$ (Nagelkerke $R 2$ ) of the variance in atrophic gastritis or intestinal metaplasia and correctly classified $86.3,85.3 \%$ of cases respectively. Smokers were 36.72 and 23.73 times more likely to exhibit atrophic gastritis or intestinal metaplasia. Previous H. Pylori was associated with an increased likelihood of exhibiting atrophic gastritis. Also increasing WBCs was associated with an increased likelihood of exhibiting atrophic gastritis or intestinal metaplasia.

$85.3 \%$ of cases, respectively. Smokers were 36.72 and 23.73 times more likely to exhibit atrophic gastritis or intestinal metaplasia. Previous H. Pylori was associated with an increased likelihood of exhibiting atrophic gastritis. Also increasing WBCs was associated with an increased likelihood of exhibiting AG or IM. However, age, sex, and hypertension haven't shown any statistical significance (Table 5).

Etiologically, H. pylori infection prior to endoscopy was tested using $H$. pylori-Ag in stool by ELISA ; chronic gastritis, AG, and IM were statistically significant among patients who were positive than negative for $H$. pylori infection. Additionally, when we compared the 3 groups regarding previous therapy for $H$. pylori, we found no statistically significant difference between the groups. Surprisingly, a portion of patients who had completed their anti-H. pylori therapy was complicated by chronic gastritis, AG or IM raising the question of progression of gastric inflammatory process post-treatment or there was unsuccessful eradication (Table 4).

On general examination of the total sample, Pallor was the most noticeable sign in 29 patients $(19.3 \%)$ of the total sample with HB mean \pm SD was $(10.57 \pm 1.63)$ (Table 1 \& 4). As regards blood indices, HB, WBCs, and Platelets were significant between GI and GII, p-values $(0.001,0.05,0.05)$, respectively and IM showed the lowest HB level mean \pm SD $(9.30 \pm 0.90)$ (Table 4). Persistent abdominal pain and dyspepsia were the most common indications for EGD 122 patients $(81.3 \%) \&$ $75(50 \%)$, respectively. Other manifestations include vomiting, heartburn, unexplained anemia, GI bleeding, and dysphagia (Table 1).

According to the sites where biopsies were taken from the stomach, antral biopsies were the commonest
(92, 61.3\%), fundal $(35,23.3 \%)$, and finally corpus $(23,15.3 \%)$. Glandular atrophy and IM were not significant statistically between antral, corpus, or fundus biopsies (p-value $0.37 \& 0.19$ ) (Table 1).

We correlated the standardized endoscopic findings in each group, diffuse redness was more common in GII $45.8 \%$, mottling in GI $(74.3 \%)$, visible submucosal vessels, atrophic mucosa and/or gastric folds predominated in GII $(75.6,82.3 \& 73.1)$, respectively. Whitish raised lesions were more specific in GIII (85.7\%) with a highly statistically significant difference between the groups. H. pylori-induced pathology revealed the most common etiology in all groups $132 / 150$ patients $(88 \%)$ (p-0.001). Bile reflux-induced gastritis was an additional cofactor in 21 cases $(14 \%)$ with no identifiable cause in 18 cases $(12 \%)$ (Table $2 \&$ Figure 4$)$.

Histopathological distribution according to the updated Sydney system revealed $H$. pylori intensity was a statistically significant difference between the 3 groups (p-value 0.001) with moderate intensity in GI \&GIII and mild intensity in GII (Table 2).

According to the above criteria for each group, the sensitivity and specificity of standard EGD regarding the diagnosis of chronic gastritis and AG were high ranging from $85-88 \%$ with a good degree of agreement (0.7-0.71) $(\mathrm{P}<0.001)$. In contrast, the sensitivity yield of diagnosing IM was very low $54 \%$ and specificity was $100 \%$ giving the satisfaction that EGD can be a good positive tool regarding the diagnosis of IM (Table 3).

In comparing the ability of CWLE to suspect chronic gastritis, AG and IM with the proven-biopsy, results were $55(36.7), 81(54 \%), 14(9.3 \%)$ and the HP results were $42(28 \%), 82(54.7 \%), 26(17.3 \%)$, respectively. In other words, the standard EGD could truly diagnose 36 out 
of 42 patients diagnosed by HP in GI, 71/82 in GII, and 14/26 in GIII. In 42 cases proved by HP to have chronic gastritis, 36 were endoscopically suspected as chronic gastritis, 8 cases were AG with no cases were suspected with IM. Among the 82 cases with AG ; 71 patients from AG and 11 only were chronic gastritis. IM was detected in 26 patients (4 with suspected chronic gastritis, 8 suspected to have AG and 14 were highly predicted to have IM endoscopically). Cases done with endoscopies that have NBI were statistically significant difference than cases done with standard scopes ( $p$-value $<0.001)$ (Figure 3).

\section{Discussion}

The aging process nowadays has increased the incidence of GC despite early detection of AG, IM, and successful eradication of $H$. pylori which constitutes the most common etiology of chronic gastric inflammation, which if not treated will develop AG and metaplastic changes and finally GC (11). In Egypt, there is a paucity of data documenting the incidence of AG and IM among patients either actively or previously infected with $H$. pylori. Additionally, due to limited resources in many centers, we still rely on HP diagnosis of AG and IM which may not be accurate due to poor endoscopic resources and consequently ill-defined biopsy mapping.

In the current study, the incidence of previous $H$. pylori infection was increased with AG (P 0.01, OR 4.5) on multivariate analysis and a trend towards increased abdominal pain $(122,81.3 \%)$ and dyspepsia $(75,50 \%)$ in all groups. Additionally, there was a statistically significant difference among the groups with aa higher incidence of $\mathrm{AG}$ and IM in patients who had a positive family history of $H$. pylori infection ( $p$-value $<0.001$ ). However, among risk factors for IM in Gomez et al., there was a statistically significant difference between weight loss and IM (P -0.01 OR 0.07) (3).

Among the most common indications for EGD in the current project patients' with gastric IM were abdominal pain and weight loss $(41.7 \% \& 13.5 \%)$ on univariate analysis, $\mathrm{P}$ values for abdominal pain and weight loss were (P - $0.005 \& 0.01)$, respectively with a trend towards increased the frequency of gastric IM(GIM) [OR 1.81 $(0.95,3.66) \mathrm{P}=0.073]$.

Anemia deemed to be significant in GIII (P-0.001) probably due to vomiting, dyspepsia, $H$. pylori infection and gastric erosions and/or ulcers that may cause chronic blood loss.

In our research, there was a trend on multivariate analysis towards increased frequency of smoking ( $\mathrm{P}$ $<0.01$, OR 23.73) and increasing WBCs ( $<<0.02$, OR 0.5 ) among patients with IM. Gomez et al. proved a statistically significant difference between 468 patients with GIM and 118 without GIM as regards age, a smoking lifetime without difference regarding positive family history of cancer stomach and gender. Patients with GIM had older ages and a longer duration of smoking than a group without GIM on univariate analysis $(\mathrm{P}<0.001 \& 0.007)$, respectively. Moreover, multivariate analysis for tobacco use had an odds ratio (OR) 1.73(CI $1.18,2.55) \mathrm{p}=0.073(3)$.

In Gomez et al., there was no statistically significant difference between positive family history of GC and the development of IM (P-0.5, OR-1.38) (3) ; our study consisted with these results with the family history of GC in the total sample was $10 / 150(6.7 \%)$ patients, but not related to the development of $A G$ and $\operatorname{IM}(p>0.05)$. This result was proved in a meta-analysis that described (OR) of 1.982 for the presence of GIM in first-degree relatives of GC patients (13).

Most of the studies estimated the incidence of IM in patients underwent EGD and had biopsies to be less than $10 \%$ (3). In our study, the incidence of IM reported $17.3 \%$ may be due to late detection and lack of routine cancer screening programs.

Sydney system recommends taking five gastric specimens to diagnose $H$. pylori infection and gastric mucosal abnormalities, albeit in daily practice it seems to be impractical to have five biopsies with higher costs and overload for histopathologists especially with a revolutionary advance in magnifying endoscopies (11). A recent study from Thailand in 2015 done on 500 patients complaining of dyspeptic symptoms, EGD has done with a site-specific biopsy technique to detect H.pylori infection and premalignant conditions, it yielded a good efficacy than a standard biopsy with sensitivity, specificity, positive and negative predictive values using standard endoscopies to detect H.pylori infection were $(95.4 \%, 97.3 \%, 98.8 \%, 90 \%)$, respectively with $\mathrm{P}<0.01(11)$.

Many hypotheses reported a strong correlation between H. pylori and the development of AG and IM. After eradication of $H$. pylori, the regression of these lesions occurs. Several meta-analyses reported the decreased occurrence in GC by $33 \%$ in $H$. pylori-successfully eradicated cases. However, eradication after developing gastric atrophy and IM remains unclear (12). Gomez et al. showed a potent significant correlation between H. pylori and IM ( $\mathrm{p}=0.007$ OR 3.07) (3). In our study, H. Pylori infection was associated with an increased likelihood of exhibiting AG ( $\mathrm{p}=0.01$ \& OR 4.49) and IM $p=0.09 \&$ OR 4.6. However, a large meta-analysis by Wang and his colleagues involved 12 studies with more than 2500 patients having AG and IM, concluded that antral AG could regress with treatment while corpus AG and gastric IM failed to regress despite eradication therapy, augmenting the theory that, IM is a breakpoint for cancer stomach (10).

AG is generally underestimated especially mild AG using standard CWLE by the visibility of submucosal vessels and increasing gastric mucosal whitish areas. However, by using magnifying endoscopy (ME) techniques, mild cases may be easily determined (12).

In a previous study comparing CWLE with Blue light imaging, revealed a detection rate of $3.3 \%$ for early GC 
after $H$. pylori eradication therapy. (11) We conclude from our study and others', that H.pylori therapy may not prevent the progression of chronic and AG to IM and dysplasia denoting that anti-H.pylori treatment should not give a high sense of safety for patients and follow up is recommended.

The most common endoscopic findings in a total sample population with and without IM in Gomez et al. study were chronic inflammation $(137 / 589,23.3 \%)$ and gastric nodularity $(104,17.7 \%)(3)$. In correlation with our study, the incidence of diffuse redness in all groups was $(96,64 \%)$, visible submucosal vessels $(41 / 150)$ with a trend towards increased frequency in AG and highly statistically significant difference between the groups ( $p$ $<0.001$ ), whitish raised plaques were more frequent in IM group 85.7\% (12/26, p-<0.001) (Table 2).

Previous well-validated studies using CWLE documented the poor correlation between endoscopic and histopathological findings for the detection of precancerous gastric lesions. On the contrary, recent trials using high-definition WLE (HD-WLE) showed promising outcomes. A cross-sectional study reported overall accuracy $88 \%$ using HD-WLE for detection of IM and dysplasia with a sensitivity of $75 \%$ and specificity of $94 \%$. Another prospective multicenter study showed a $59 \%$ sensitivity and $98 \%$ specificity for IM (12).

In a study done by Jian-Min et al. 35 out of 140 cases were diagnosed by HP to have chronic AG, only 8 by CWLE had features of AG but with ME, 33 were detected with a statistically significant difference between the two scopes $p=$ value $<0.01(14)$. In this project, the sensitivity and specificity of detecting AG using CWLE were $87 \% \&$ $85 \%$, respectively and ROC/AUC was 0.85 .

In another study by Fukuta and others in 2013, on 163 Japanese patients indicated for EGD and biopsies were taken according to the Sydney system, $46.6 \%$ had chronic gastritis, and $16.6 \%$ had early GC by CWLE. The sensitivity, specificity, and ROC/AUC for detection of IM in gastric antrum using the standard endoscopy were $94.6 \%, 69.1 \%$, and 0.82 , respectively (15). In this project, the sensitivity, specificity, and ROC/AUC of IM using CWLE were $54 \%, 100 \%$, and 0.77 , respectively. (Figure 2).

Among the most frequent endoscopic findings in Gomez et al. research, were gastritis $23.3 \%$, AG 10.8\% of all patients and in $13.2 \%$ of patients with GIM ; while, after HP analysis, chronic gastritis was confirmed in $65.6 \%$ of GIM group and $32.3 \%$ in the group without GIM. $H$. pylori infection was detected by HP in 46/486(9.8\%) in GIM and 6/171(3.5\%) in non-GIM group. Multivariate analyses were [OR $3.07(1.33,8.20), \mathrm{P}=0.007$ ] (3).

An Indian study in 2010 compared the diagnostic sensitivity of CWLE versus NBI for the detection of premalignant gastric lesions, a total of 200 patients were examined using both scopes randomly. CWLE was able to detect AG and IM in 17 patients and 31 using NBI (p$0.001)$ (16).
A different data elaborated in a screening study done to compare two protocols for the screening of gastritis and IM whereby the first protocol used the HD-WLE with biopsies taken from the stomach according to the updated Sydney system and targeted biopsies when needed and the second was using NBI and targeted biopsies from any suspicious premalignant lesion. Results revealed,92 out of 119 patients had chronic gastritis versus $71 / 119$ (p 0.0001 ), AG $32.7 \%$ versus $23.5 \%$ (p0.03) and IM 16\% versus $15.1 \%(\mathrm{p}-0.7)$ by HD-WLE and NBI, respectively for each group (17).

In this study, the sensitivity and specificity for IM using NBI were $100 \& 96 \%$, respectively and for AG were $87 \& 93 \%$. But, with conventional WLE were $86 \%$ \& $88 \%$ for GI with accuracy $87 \%, 87 \% \& 85 \%$ for GII with accuracy $86 \%$ and for GIII $54 \%$ \& $100 \%$, respectively and accuracy $92 \%$. So, NBI increased the diagnostic accuracy of chronic gastritis, IM and AG by 8,2 , and $4 \%$ than CWLE $(\mathrm{p}<0.001)$ with a highly statistically significant difference between the two scopes (Table 3).

To conclude, CWLE has high sensitivity and specificity for suspicion of chronic gastritis and AG, but low sensitivity and very high specificity for IM. Targeted biopsies with image enhanced techniques may be more practical than updated Sydney protocol, especially in a high-risk population. But, in developing and low-risk countries, we can still depend on CWLE and HD-WLE, obtaining biopsies according to the updated Sydney system and targeted biopsies from macroscopic lesions with comparable results with NBI and targeted biopsies.

\section{No conflict of interest, no funding source for all authors.}

\section{References}

1. FUKUTA N., IDA K., KATO T., UEDO N., ANDO T., WATANABE H., et al. Endoscopic diagnosis of gastric intestinal metaplasia: a prospective multicenter study. Dig. Endosc., 2013, 25(5) : 526-534. doi : 10.1111/ den. 12032

2. COSMINA D., ELENA M., DANIELA P., OLGA B., MARIA O., ALINA B., et al. Gastric Intestinal Metaplasia : Prevalence, Clinical Presentation, Endoscopic and Histological Features. Acta Medica Marisiensis, 2016, 62(1) : 56-59.

3. GOMEZ JM., PATRIE JT., BLEIBEL W., FRYE J., SAUER B., SHAMI $\mathrm{V}$., et al. Gastric intestinal metaplasia is associated with gastric dysplasia but is inversely correlated with esophageal dysplasia. World J. Gastrointest. Endosc., 2017, 9(2) : 61-69.

4. TSUKAMOTO T., NAKAGAWA M., KIRIYAMA Y., TOYODA T., CAO X. Prevention of Gastric Cancer : Eradication of Helicobacter Pylori and Beyond. Int. J. Mol. Sci., 2017; 18.

5. LEUNG WK., WONG IOL., CHEUNG KS., YEUNG KF., CHAN EW., WONG AY. et al. Effects of Helicobacter pylori Treatment on Incidence of Gastric Cancer in Older Individuals. Gastroenterology, 2018, 155(1) : 67-75. doi : 10.1053/j.gastro.2018.03.028

6. LAHNER E., CARABOTTI M., ESPOSITO G., HASSAN C., ZULLO A., ANNIBALE B., et al. Occurrence and predictors of metaplastic atrophic gastritis in a nation-wide consecutive endoscopic population presenting with upper gastrointestinal symptoms. Eur. J. Gastroenterol. Hepatol., 2018, 30(11) : 1291-1296. doi : 10.1097/MEG.0000000000001246

7. KATO M. Endoscopic Findings of H. pylori Infection. In : Suzuki H., Warren R., Marshall B. (eds) Helicobacter pylori. Springer, Tokyo, 2016.

8. EZOE Y., MUTO M., UEdO N., Doyama H., Yao K., Oda I., et al. Magnifying narrowband imaging is more accurate than conventional white- 
light imaging in diagnosis of gastric mucosal cancer. Gastroenterology, 2011, 141(6) : 2017-2025.e3. doi : 10.1053/j.gastro.2011.08.007

9. BISSCHOPS R., AREIA M., CORON E., DOBRU D., KASKAS B., KUVAEV R., et al. Performance measures for upper gastrointestinal endoscopy: A European Society of Gastrointestinal Endoscopy quality improvement initiative. United European Gastroenterol. J., 2016, 4(5) : 629656. doi : 10.1177/2050640616664843

10. Wang J., Xu L., Shi R., HUANG X., LI SW., HUANG Z, ZHANG G. et al. Gastric atrophy and intestinal metaplasia before and after Helicobacter pylori eradication : a meta-analysis. Digestion., 2011, 83(4) : 253-260. doi : $10.1159 / 000280318$

11. TONGTAWEE T., KAEWPITOON S., KAEWPITOON N., DECHSUKHUM C., LEEANANSAKSIRI W., LOYD RA., et al. Diagnosis of Helicobacte pylori Infection. Asian Pac. J. Cancer Prev., 2016, 17(4) : 1631-1635. doi : 10.7314/apjcp.2016.17.4.1631

12. PIMENTEL-NUNES P., LIBÂNIO D., MARCOS-PINTO R., MI GUEL A., MARCIS L., GIANLUCA E., et al. Management of epithelial precancerous conditions and lesions in the stomach (MAPS II) : European Society of Gastrointestinal Endoscopy (ESGE), European Helicobacter and Microbiota Study Group (EHMSG), European Society of Pathology (ESP), and Sociedade Portuguesa de Endoscopia Digestiva (SPED) guideline update 2019. Endoscopy, 2019, 51(4) : 365-388.
13. DOHI O., YAGI N., NAITO Y., FUKUI A., GEN Y., IWAI N., et al. Blue laser imaging-bright improves the real-time detection rate of early gastric cancer : a randomized controlled study. Gastrointest. Endosc., 2019, 89(1): 47-57. doi : 10.1016/j.gie.2018.08.049

14. JIAN-MIN Y., LEI C., YU-LIN F., XIANG-HONG L., XIN Y., DIAN-CHUN F., et al. Endoscopic patterns of gastric mucosa and its clinicopathological significance. World J. Gastroenterol., 2013, 9(11) : 2552-2556.

15. FUKUTA N., IDA K., KATO T., UEDO N., ANDO T., WATANABE H., et al. Endoscopic diagnosis of gastric intestinal metaplasia : a prospective multicenter study. Dig. Endosc., 2013, 25(5) : 526-534. doi : 10.1111/ den. 12032

16. CHACKO A., DUTTA A., SAJITH KG. Narrow Band Imaging vs. White Light Gastroscopy in Detecting Gastric Premalignant and Early Malignant Lesions: A Randomized Prospective Crossover Study. Gastrointestinal Endoscopy, 2010, 71(5), AB144-AB145, doi.org/10.1016/j.gie.2010.03.1056.

17. XIROUCHAKIS E., LAOUDI F., TSARTSALI L., SPILIADI $C$, GEORGOPOULOS SD. Screening for gastric premalignant lesions with narrow band imaging, white light and updated Sydney protocol or both? Dig. Dis. Sci., 2013, 58(4) : 1084-1090. doi : 10.1007/s10620-012-2431-x 